EPJ Web of Conferences 88, 01011 (2015)

DOI: $10.1051 /$ epjconf/ 20158801011

(C) Owned by the authors, published by EDP Sciences - SIF, 2015

\title{
GLORIA: A compact detector system for studying heavy-ion reactions using radioactive beams
}

\author{
G. Marquínez-Durán ${ }^{1}$, L. Acosta ${ }^{1,2}$, R. Berjillos ${ }^{1}$, J.A. Dueñas ${ }^{1}$, \\ J.A. Labrador ${ }^{3}$, K. RuseK ${ }^{4}$, A.M. SÁnchez-Benítez ${ }^{1,5}$ and \\ I. MARTEL ${ }^{1}$ \\ ${ }^{1}$ University of Huelva, Huelva, Spain \\ 2 INFN-Laboratori Nazionali del Sud, Catania, Italy \\ ${ }^{3} \mathrm{CNA}$, Seville, Spain \\ ${ }^{4}$ University of Warsaw, Warsaw, Poland \\ ${ }^{5}$ Centro de Física Nuclear da Universidade de Lisboa (CFNUL), Lisbon, \\ Portugal
}

\begin{abstract}
The GLObal ReactIon Array (GLORIA) has been designed in order to study direct nuclear reactions induced by exotic nuclei at energies close to the Coulomb barrier. The detector array consists of six silicon particle-telescopes arranged in a very close geometry around a $30^{\circ}$ rotated-target system, allowing the measurement of reaction fragments in a continuous angular range from $15^{\circ}$ to $165^{\circ}$ (Lab.). GLORIA has been used for the first time at the SPIRAL/GANIL facility at Caen (France) to study the scattering of the system ${ }^{8} \mathrm{He}+{ }^{208} \mathrm{~Pb}$ at the collision energies of 16 and $22 \mathrm{MeV}$.
\end{abstract}

\section{Introduction}

Large solid angle detectors with high efficient particle identification capabilities are usually required when using low intensity radioactive beams. Silicon detectors present excellent characteristics for detecting heavy ions at moderate counting rates with high detection efficiency and good energy resolution. For these reasons, they have become commonly used devices for the construction of charged particle detector arrays. Sometimes, in the analysis of experiments in which particles telescopes are used, several difficulties are 
found, as the lack of symmetry between them or an absence of overlapping in the covered angular regions, complicating the measurement in a continuous range and the comparison between counting rates in different detectors. Usually, every telescope is mounted independently so individual movements can occur hindering the assignment of solid and scattering angles. The aim of GLORIA [1] is to eliminate, as far as possible, these disadvantages.

\section{Design of the detector array}

Some requirements were imposed during the process of design as a continuous angular coverage between $15^{\circ}$ and $165^{\circ}$, an angular overlapping between adjacent telescopes of at least $10^{\circ}$ and a reflection symmetry of the configuration in order to reduce systematic uncertainties. The final configuration of GLORIA consists in 6 particle telescopes covering, with no gaps, a continous angular range between $15^{\circ}$ and $165^{\circ}$ (Lab.) with an overall solid angle of $26.1 \%$ of $4 \pi$. Each of them is made of a first $40 \mu \mathrm{m}$ Double-Sided Silicon Strip Detector (DSSSD) and a second $1 \mathrm{~mm}$ DSSSD. Two telescopes are located forward $\left(15^{\circ}\right.$ and $\left.62^{\circ}\right)$, two backward $\left(117^{\circ}-165^{\circ}\right)$ one on top $\left(82^{\circ}\right.$ $\left.128^{\circ}\right)$ and one bottom $\left(52^{\circ}-97^{\circ}\right)$. The target is introduced $30^{\circ}$ rotated with respect to the vertical plane avoiding the shading of detectors.

\section{Monte Carlo simulations}

Monte Carlo simulations have been performed by means of Geant4 [2] and the NPTool [3] packages. The complete geometry of GLORIA, including

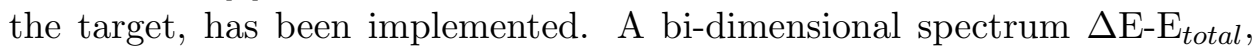
obtained from the simulations, for the central pixel of a forward telescope at $21^{\circ}$ is shown in figure 1 (left). The banana-like regions correspond to various test isotopes from ${ }^{9} \mathrm{Be}$ to ${ }^{13} \mathrm{C}$. For evaluating the quality of the mass discrimination achieved, the value of the Figure of Merit (FoM) has been studied for the case of the carbon isotopes. For this, a projection of the energy slice marked in figure 1 (left) with a red square has been projected onto the $\Delta \mathrm{E}$ axis, and the result is shown in figure 1 (right). The obtained distribution has been analysed using a Gaussian fit and a value of $\mathrm{FoM}=0.9$ for the ${ }^{12} \mathrm{C} /{ }^{13} \mathrm{C}$ has been obtained. A detector system will exhibit good identification capability when $\mathrm{FoM} \geq 0.7[6]$. 

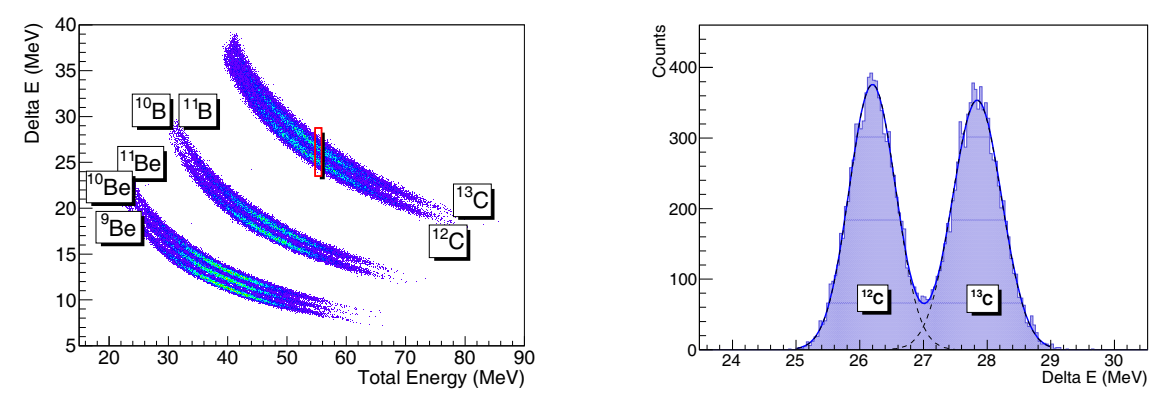

Figure 1: (Left) Simulated mass spectrum obtained at the central pixel of a forward telescope. (Right) Projection of an energy slice of the carbon mass region onto the Delta E axis, in order to evaluate the FoM (see text for details).

\section{First experimental results with exotic beams}

The GLORIA detection system was commissioned in October 2010 in the SPIRAL ISOL facility at GANIL. A radioactive ion beam of ${ }^{8} \mathrm{He}$ at energies of 16 and $22 \mathrm{MeV}$ was used to study the dynamics of the system ${ }^{8} \mathrm{He}+{ }^{208} \mathrm{~Pb}$ at energies around the Coulomb barrier. Preliminary results can be found at $[4,5]$. A preliminary angular distribution of the elastic cross section is shown in figure 2, where data points obtained from the six telescopes are plotted with different colours. Scattering angles were obtained by using Monte Carlo simulations, taking the centre of the corresponding ion distribution registered at each single pixel. Good matching is found between the six telescopes of GLORIA, providing a smooth and continuous angular distribution.

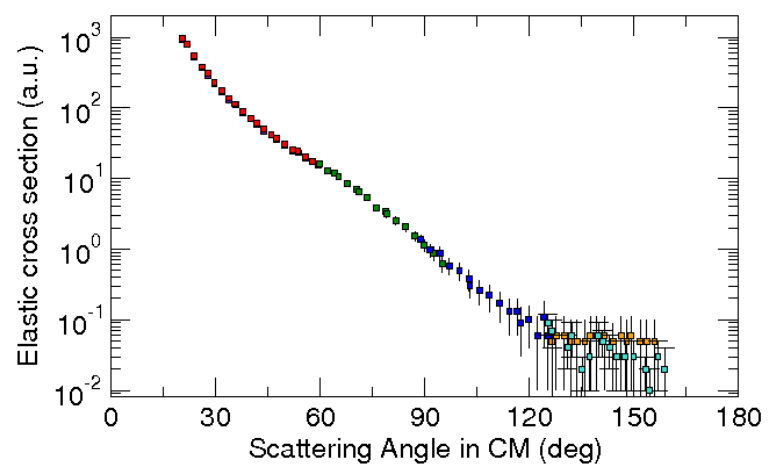

Figure 2: Preliminary angular distribution of elastic cross-sections (arbitrary units). Every telescope is plotted with a different colour. 
In figure 3 the total energy of the events in the mass region corresponding to ${ }^{6} \mathrm{He}$ and ${ }^{4} \mathrm{He}$ isotopes are plotted vs. the observation angle. Regions attributed to neutron transfer to the continuum have been surrounded with a black solid line. With a red curve, the region which has been identified as incomplete charge collection of ${ }^{8} \mathrm{He}[1]$ is marked.
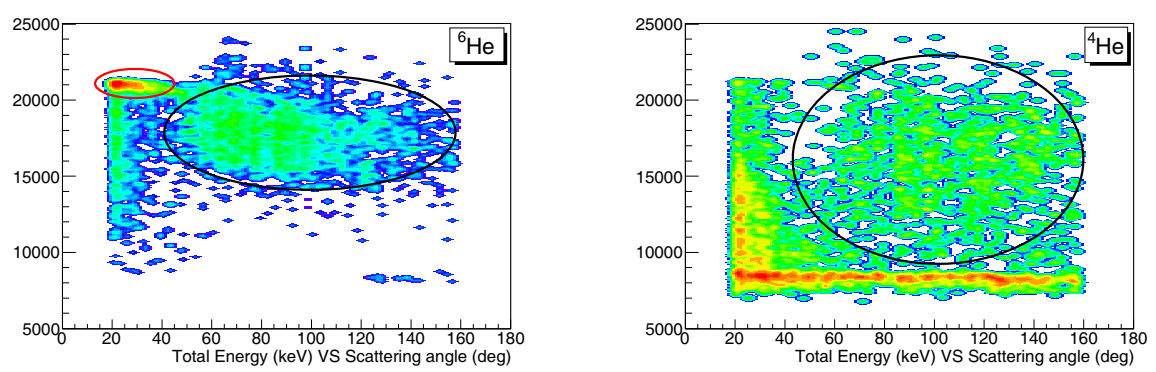

Figure 3: Plot of energy vs. observation angle for events in the mass region corresponding to ${ }^{6} \mathrm{He}$ (left) and ${ }^{4} \mathrm{He}$ (right) for the full GLORIA array.

\section{Acknowledgements}

This work was supported in part by the Grant FPA2010-22131-C02-01 (FINURA) from the Spanish Ministry of Economy and Competitiveness, the Grant N202 033637 from the Ministry of Science and Higher Education of Poland and the Contract EUI2009-04163 (EUROGENESIS) from the European Science Foundation.

\section{References}

[1] G. Marquínez-Durán et al., Nucl. Instr. and Meth. A 755, 69-77 (2014)

[2] http://geant4.cern.ch

[3] http://gaspard.in2p3.fr/simu.html

[4] G. Marquínez-Durán et al., Act. Phys. Pol. 43, 239 (2012)

[5] G. Marquínez-Durán et al., Act. Phys. Pol. 44, 467 (2013)

[6] R.A. Winyard et al., Nucl. Instr. and Meth. A 95, 141 (1971) 